\title{
Joint INTEGRAL and AGILE observations of FSRQs
}

\author{
I. Donnarumma*, L. Pacciani, V. Vittorini, F. D’Ammando, G. Pucella, A. De Rosa, \\ M.T. Fiocchi, M. Tavani, P. Ubertini \\ INAF/IASF Roma, via Fosso del Cavaliere 100, 00133, Rome, Italy \\ E-mail: immacolata.donnarumma@iasf-roma.inaf.it
}

\section{S. Vercellone, A. Chen, A. Giuliani}

INAF/IASF Milano, Via E. Bassini, 15 - 20133 Milano, Italy

\section{A. Bulgarelli}

INAF/IASF Bologna, Via Gobetti 101, I-40129 Bologna, Italy

\section{F. Longo}

Dip. di Fisica and INFN-Trieste, Via Valerio 2, I-34127 Trieste, Italy

During the first year of observations, AGILE detected several blazars, most of them Flat Spectrum Radio Quasars. For a subset of this sample, extensive multi-frequency campaigns were carried out. We report on three FSRQs for which joint AGILE and INTEGRAL observations were requested and obtained: 3C 454.3, 3C 273 and PKS 1830-211. 3C 454.3 is the most active blazar detected by AGILE. It was observed in $\gamma$-ray during July 2007, November-December 2007, MayJune 2008, July-August 2008. In November 2007 an INTEGRAL pre-approved AO5 ToO was also activated. As opposite to the gamma-rays, the source was in low state in the hard X-rays. The high gamma-ray state of the source also triggered a Swift ToO at soft X-rays. 3C 273 was observed on December 2007 during coordinated INTEGRAL-AGILE observations planned as open time for INTEGRAL AO5. The source was found to be in high state both in the gammarays and hard X-rays. Also in this case a Swift ToO was activated to monitor the soft X-ray behaviour of the source. Despite of the deep coverage of the galactic plane, PKS 1830-211 was not detected by AGILE on daily or weekly time scales. By accumulating all the available AGILE data, we can provide a deep upper limit $\left(12 \times 10^{-8} \mathrm{ph} \mathrm{cm}^{-2} \mathrm{~s}^{-1}\right)$ for this source at energies above $100 \mathrm{MeV}$. PKS 1830-211 was extensively observed also by INTEGRAL as a part of AO5 Key Programme, thus providing an unprecedented gamma and hard X-ray monitoring over one year time scale. These data allowed us to investigate possible correlations between the two energy ranges. Moreover, the large multi-frequency coverage involving also radio and optical monitoring (WEBT and REM) of these sources gave us the opportunity to investigate their Spectral Energy Distributions (SEDs) from optical up to gamma-ray energy bands, studying both the synchrotron and inverse Compton emissions. In particular we found that the 3 SEDs are well fitted by a one zone Synchrotron Self Compton (SSC) model plus an External Compton (EC) component which seed photons come from the accretion disk and/or the Broad Line Region (BLR).

7th INTEGRAL Workshop

September 8-11 2008

Copenhagen, Denmark 


\section{Introduction}

Blazars are a subclass of Active Galactic Nuclei (AGN) characterized by a strong emission of non-thermal radiation across the entire electromagnetic spectrum, from radio to very high $\gamma$-ray energies (Ghisellini et al. 1998; Fossati et al. 1998). The typical observational properties include irregular, rapid and often very large variability, apparent super-luminal motion of the radio jet components, flat radio spectrum, high and variable polarization at radio and optical frequencies. Their emission properties, extending on several decades in energy, make multi-wavelength campaigns the key to understand the physical mechanisms responsible for the observed SED. The SEDs of these objects are typically double-humped with a first peak occurring in the IR/Optical band for the so-called red blazars (including Flat Spectrum Radio Quasars [FSRQs] and Low-energy peaked BL Lacs) and at UV/X-rays for the so-called blue blazars (including High-energy peaked BL Lacs). Moreover, long term monitoring of these sources lasting several weeks is mandatory to study their unpredictable duty cycle. The AGILE satellite (Tavani et al. 2008) launched on 2007 April 23, with its pointing strategy lasting 2-3 weeks, gives good chances to monitor the variability of these sources by means of multi-frequency observations. The deep coverage of selected regions has allowed us to study the daily variability of brighter sources (Vercellone et al. 2008, 2009) and also to provide significant detection or strict upper limits in the case of fainter sources.

In the following sections we will report on three FSRQs observed simultaneously by AGILE and INTEGRAL during 2007-2008: 3C 454.3, 3C 273 and PKS 1830-211.

\section{The multi- $\lambda$ light curves}

\section{$2.13 \mathrm{C} 454.3$}

3C 454.3 is a FSRQ at redshift $z=0.859$, one of the brightest extragalactic radio sources displaying super-luminal motion of its radio jet. It is a highly variable blazar, showing outburst on time scales of years in the $\mathrm{cm}$ to $\mathrm{mm}$ regime, few days in optical and $\gamma$-ray energy bands (Raiteri et al. 2008, Vercellone et al. 2008, 2009). On the other hand, it is less clear the behaviour of its hard X-ray emission during the observed optical and $\gamma$-ray high states. AGILE and Fermi opened a new era in the study of blazars. In particular, joint hard X-ray and gamma-ray observations were performed by INTEGRAL and AGILE during a multi-wavelength campaign in November 2007.

AGILE data were collected during 2 periods, the first between 2007-11-10 and 2007-11-25 and the second between 2007-11-28 and 2007-12-01, for a total of $592 \mathrm{ks}$. INTEGRAL data were collected during a pre-approved AO5 ToO on revolutions 623 and 624 (300 ks). A Swift ToO monitored 3C 454.3 for a total exposure of $10 \mathrm{ks}$. The optical monitoring during the AGILE observations was performed by both WEBT and REM. 3C 454.3 showed high $\gamma$-ray activity, with flares on a time scale of few days and an average flux $F(E>100 \mathrm{MeV})=170 \times 10^{-8} \mathrm{ph} \mathrm{cm}^{-2}$ $\mathrm{s}^{-1}$. The R-optical band also showed strong variability on intra-day time scale (see Fig. 1a). A Discrete Correlation Function performed between $\gamma$-rays and optical emissions seems to show no time-lag in the correlated variability. On the other hand, both the Swift/XRT (Burrows et al. 2005) light curve in $0.3-10 \mathrm{keV}$ and the INTEGRAL/IBIS (Ubertini et al. 2003) light curve in the energy

\footnotetext{
* Speaker.
} 
range 20-200 keV (accumulated on the whole observation) did not show statistically significant variations. We refer to Vercellone et al. 2009 for details about this multi- $\lambda$ campaign.

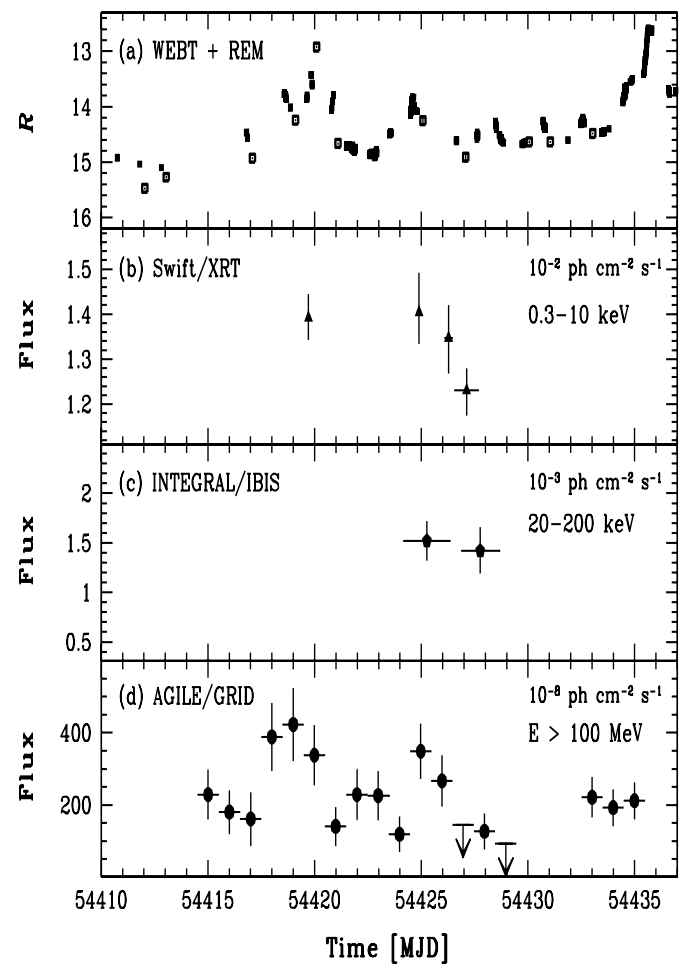

Figure 1: 3C 454.3: a) R-band optical (WEBT and REM), b)Swift/XRT, c) INTEGRAL/IBIS and d) AGILE/GRID light curves. Adapted from Vercellone et al. 2009.

\section{$2.23 \mathrm{C} 273$}

3C 273 is a very bright FSRQ, located at redshift $z=0.158$. Its SED is typical of the MeV blazars class (the IC emission peaks at a few MeV, see Sikora et al. 2002), but also shows features typical of Seyfert galaxies as broad emission lines and a big blue bump (Kataoka \& Kawai 2003). Simultaneous X-ray and $\gamma$-ray observations from $\mathrm{keV}$ to $\mathrm{MeV}$ energy range are powerful tools to investigate the IC emission, discriminating between SSC and EC models, as well as to study the dominance of the jet emission on the Seyfert-like component (Grandi \& Palumbo 2004). On the basis of these considerations, AGILE planned a multi-wavelength campaign on 3C 273 during December 2007 with simultaneous INTEGRAL observations.

3C 273 was observed by AGILE for 3 weeks between 2007 December 16 and 2008 January 8. INTEGRAL observed the source during revolutions 633 (between 2007 December 19 and December 22), 635 (between 2007 December 25 and December 28), 637 (between 2007 December 31 and 
2008 January 3). The net source exposures were 122, 580 and $494 \mathrm{ks}$ for JeM-X, ISGRI and SPI, respectively. A Swift ToO was activated with two observations between January 6 and 8 with $2.5 \mathrm{ks}$ exposure each one. The source was monitored in the optical energy bands by the REM telescope. Fig. 2 shows the multi-frequency light curve. The source exhibited $\gamma$-ray activity only in the second week of the AGILE observation. The INTEGRAL and SuperAGILE light curves show hints of anti-correlation between the hard X-ray and $\gamma$-ray activity. Moreover, we note a flux variation of $\sim 20 \%$ in the hard X-rays during the $\gamma$-ray activity (Fig. 2). On the contrary, the near-IR, optical and UV fluxes remained constant within a 10\%. We refer to Pacciani et al. 2008 for an extensive description of the data analysis (INTEGRAL/IBIS light curves in 5-20 keV and 100-200 keV are also available).

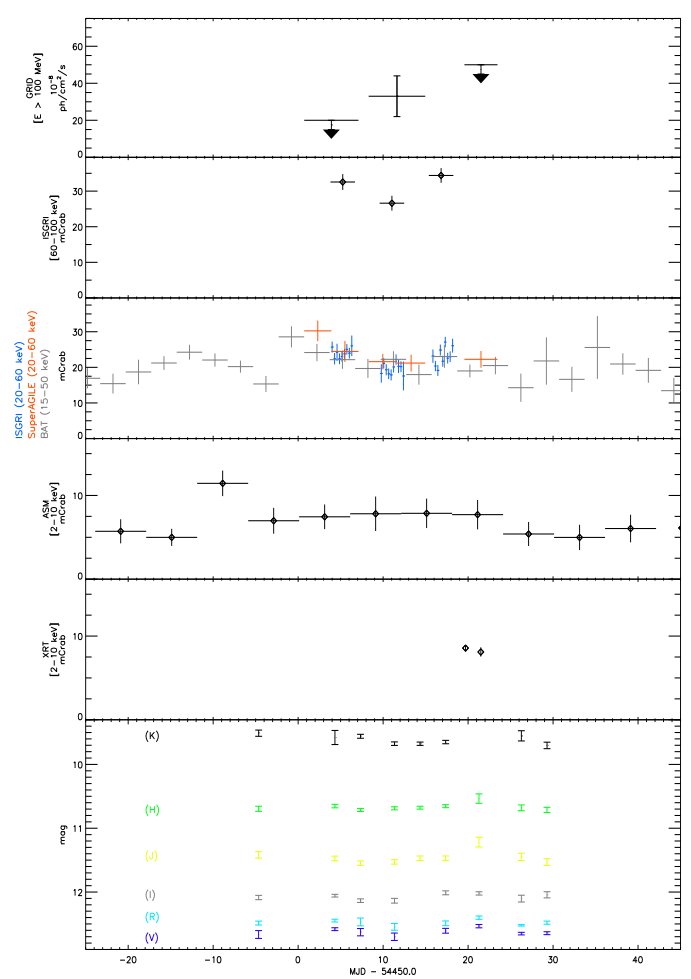

Figure 2: 3C 273. From top to bottom: the AGILE/GRID $\gamma$-ray light curve in $100-200 \mathrm{MeV}$; hard X-ray light curve from ISGRI in 60-100 keV, 20-60 keV, SuperAGILE (20-60 keV), Swift/BAT, Swift/XRT (2-10 $\mathrm{keV})$; REM light curves from V to K-band. Adapted from Pacciani et al. 2008.

\subsection{PKS 1830-211}

PKS $1830-211$ is a blazar at $\mathrm{z}=2.507$. Its radio image is gravitationally lensed by an intervening galaxy at $z=0.89$ (Wiklind \& Combes 1996) into two compact components (arising from the core) separated by 1 " and a ring-like extended structure arising perhaps from the jet. Particularly important is the absorption of the lensed images that seems to show different amount of hydrogen column densities (Dai et al. 2006). There is also evidence of variability in the absorption (Dai et al. 2008). In this work we are not focussed on the soft X-ray absorption of this source but a 
preliminary study of the SED from radio to $\gamma$-ray energy bands will be discussed.

Simultaneous hard X-ray and $\gamma$-ray data, sampling the rise and peak of the IC emission, are needed to disentangle between the source of seed photons in EC models predicted for this blazar (see De Rosa et al. 2005). INTEGRAL (Bassani et al. 2004) detected the source during the first IBIS survey (between 2003 February 28 - October 10). Several observations of this blazar were obtained as a part of AO4, AO5 and AO6 Key Programmes. Simultaneously to AO5, PKS 1830-211 was monitored in the $\gamma$-rays during two pointings towards the Galactic Center, the first between 2007-10-13 and 2007-10-24, the second between 2008-03-01 and 2008-03-30 (net on-source exposure 1.7 Ms). During these pointings AGILE did not detect the source, obtaining a $2-\sigma$ upper limit (for $E>100$ $\mathrm{MeV}$ ) of $12 \times 10^{-8} \mathrm{ph} \mathrm{cm}^{-2} \mathrm{~s}^{-1}$. For comparison EGRET detected the source during P1234 with an average flux of $(26.6 \pm 3.7) \times 10^{-8} \mathrm{ph} \mathrm{cm}^{-2} \mathrm{~s}^{-1}$ even if a lower flux $\left((17.7 \pm 3.5) \times 10^{-8} \mathrm{ph}\right.$ $\mathrm{cm}^{-2} \mathrm{~s}^{-1}$ ) was reported in the revised EGRET catalog (Casandjian \& Grenier 2008). In Fig. 3 we report the INTEGRAL/IBIS light curve in the energy range 20-40 keV accumulated between 2003 and 2008 (this includes author's private data obtained during AO4 and AO5 Key Programmes). The bin time is one month. This preliminary analysis seems to show that the source flux is roughly constant on a monthly time scale. We accumulated the spectrum on the whole period showed in Fig. 3 to improve the statistics so to extend the hard X-ray energy range up to $150 \mathrm{keV}$. The hard $\mathrm{X}$-ray data were complemented with the soft X-ray data from XMM/Newton observations (3 observations in 2004 with $30 \mathrm{ks}$ exposure each). After combining these XMM/Newton observations, we fitted simultaneously the soft and hard X-ray spectra, finding that the data were best represented by an absorbed power-law with photon index $\Gamma=1.24 \pm 0.02$. The details of the complex absorption model adopted in this work will be described in a forthcoming paper. Even if these data are not simultaneous, we underline that the stand-alone fit of the hard X-ray spectrum agrees with that obtained combining the hard and soft X-ray spectra.

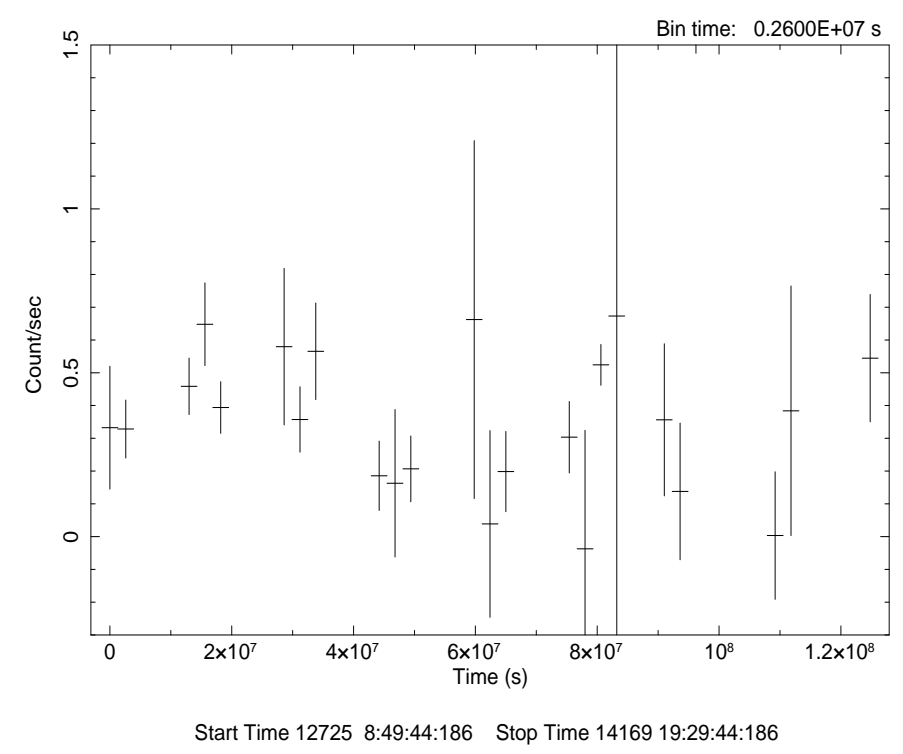

Figure 3: PKS 1830-211. INTEGRAL/ISGRI light curve in the energy range 20-40 keV between MJD 52700 and 54600 (2003-03-02 - 2008-05-14). Each bin is one month. 


\section{Discussion}

$3 C 454.3$ The SED was built up for two periods sampling two high $\gamma$-ray states as reported in Fig. 1: the first occurring between MJD 54417.5 and 54420.5 (P1), the second between MJD 54423.5 and 54426.5 (P2). Both SEDs were fitted with a one-zone leptonic model considering the contributions from SSC and from external seed photons originating both from the accretion disk and the Broad Line Region (see Vercellone et al. 2009). In Fig. 4 we report all the available multifrequency data discussed in section 2.1 complemented by radio to UV data taken from Raiteri et al. 2008. We report only the SED for P2 which contains the simultaneous INTEGRAL data, but the fit of both periods does not vary significantly. The dotted, dashed, dot-dashed and triple dot-dashed lines in Fig. 4 represent the accretion disk, the External Compton on the disk radiation, the External Compton on the BLR radiation, and the SSC contributions, respectively.

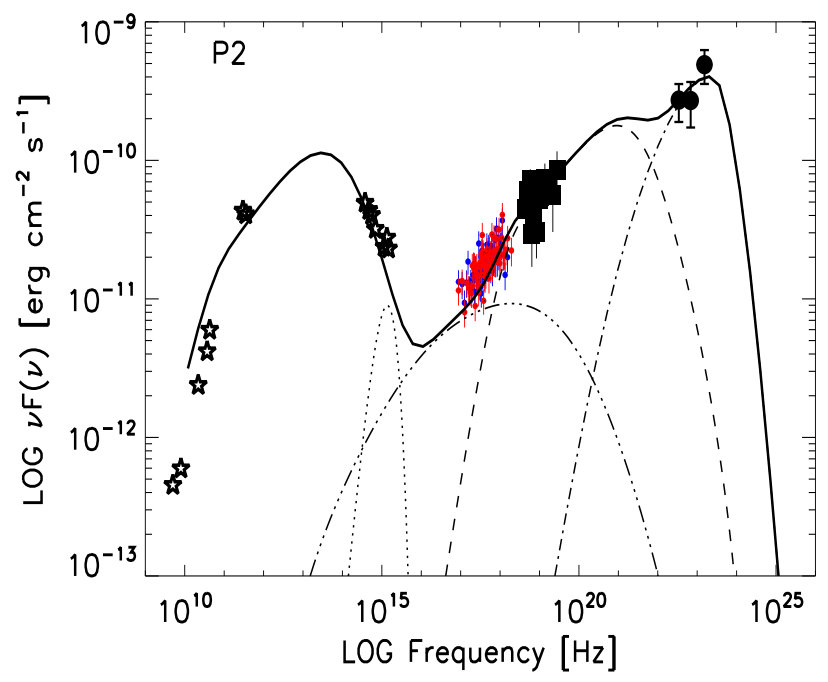

Figure 4: 3C 454.3. SED for period P2 (MJD 54423.5-54426.5). Filled circles are the AGILE data for $E>100 \mathrm{MeV}$; filled squares are INTEGRAL/IBIS data in $20-200 \mathrm{keV}$; red and blue points are Swift/XRT data; star points represent radio to UV data from Raiteri et al. 2008 corresponding to MJD 54425. Adapted rom Vercellone et al. 2009.

$3 C 273$ The SED was built up for the first and the second week of observations for which a deep AGILE upper limit and significant detection are available, respectively. The third week was neglected due to the appearance of an unidentified source close to $3 \mathrm{C} 273$, that prevented us to determine a deep upper limit. JEM-X data reported in Fig. 5 were obtained from the spectrum accumulated over all the 3 observations, but they were properly normalized to reproduce the count rates corresponding to each of the 2 observations considered here. SPI data were obtained from the average spectrum over the 3 observations. We modelled the SED with a one-zone homogeneous SSC model plus an external component from the accretion disk (dotted line in Fig. 5). The dashed line in Fig. 5 reports the SED for the first week, while the solid line represents the model for the second week. The observed variability of the SEDs cannot be associated to a synchrotron flare 
due to the lack of enhancement in all the observed wavelengths. It seems to be related to electron acceleration episode instead of jet injections.

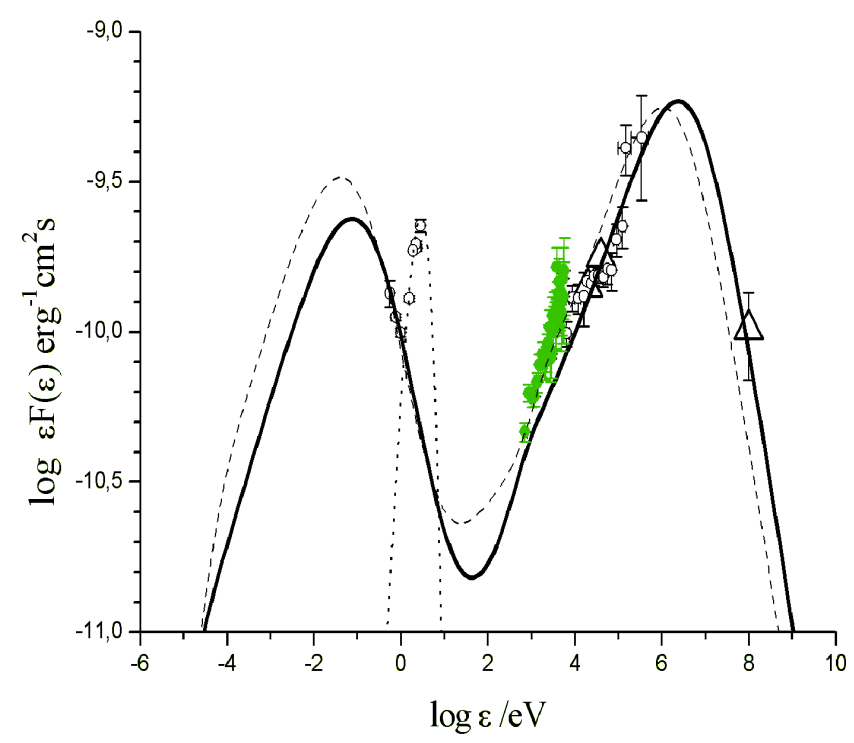

Figure 5: 3C 273. Spectral Energy Distribution obtained for the second week: AGILE data (triangular points); INTEGRAL/ISGRI and JEM-X data (open circles); Swift/XRT data (green points);Swift/UVOT and REM data. The solid line is the SED model. The model obtained for the 1st week is also present as dashed line. Adapted from Pacciani et al. 2008.

PKS 1830-211 We present the preliminary SED of PKS 1830-211 built up with XMM/Newton, INTEGRAL and AGILE data presented in section 2.2 (red points, blue triangular points and blue arrow, respectively), combined with historical radio and optical data (Rao \& Subrahmanyan 1988 and Courbin et al. 2002). The INTEGRAL spectrum was obtained integrating on the whole period between 2003 and 2008. We fitted both synchrotron and inverse Compton emissions with an EC model with soft photons coming from a disk with $L=10^{46} \mathrm{erg} \mathrm{s}^{-1}$ (solid grey line in Fig. 6). The different beaming factors for SSC $\left(\sim \delta^{4}\right)$ and EC models $\left(\sim \delta^{6}\right)$ allow for inverse Compton dominance in the SED without invoking further external sources (e.g. a torus or a BLR). We note that our conclusion could be biased by the lack of a complete set of simultaneous data. 


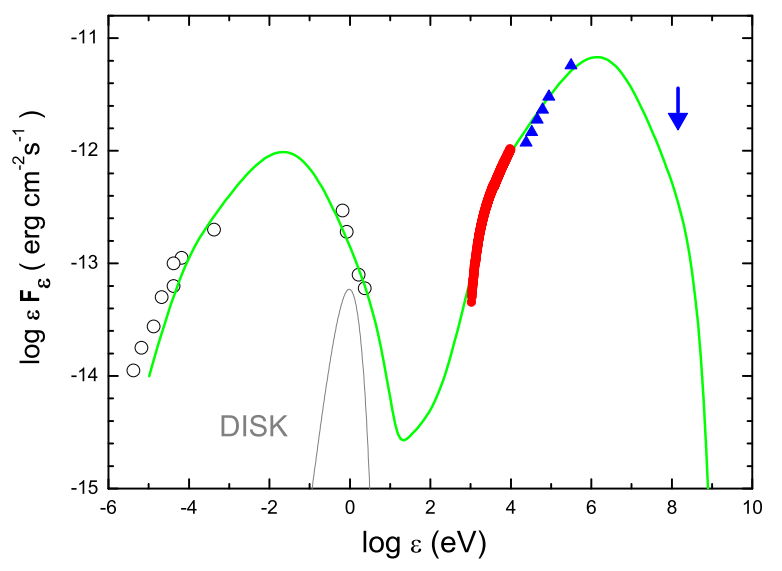

Figure 6: PKS 1830-211. Spectral Energy Distribution obtained with AGILE, INTEGRAL and XMM data (blue arrow, blue triangular points and red points, respectively). Empty circles show historical and radio optical data.

\section{References}

[1] Burrows et al.2005, Space Sci. Rev., 120, 165

[2] Casandjian \& Grenier 2008, A\&A, 489, 849

[3] Coubin et al. 2002, ApJ, 575, 95

[4] Dai et al. 2006, ApJ 637, 53

[5] Dai et al. 2008, AJ 135, 333

[6] De Rosa et al. 2005, A\&A, 438, 121

[7] Fossati et al. 1998, MNRAS 299, 433

[8] Ghisellini et al. 1998, MNRAS 301, 451

[9] Grandi, P. and Palumbo, G., 2004, Sci, 306, L998

[10] Kataoka, J., Kawai, N. 2003, NewAR, 47, 685

[11] Lovell et al. 1996, ApJ, 472, L5

[12] Pacciani et al. 2008, arXiv:0811.0593v1

[13] Raiteri et al. 2008, A\&A, 485L, 17

[14] Rao, A.P. \& Subrahmanyan, R., 1988, MNRAS 231, 229

[15] Sikora et al. 2002, ApJ, 577, 78

[16] Tavani et al. 2008, arXiv:0807.4254v1

[17] Ubertini et al. 2003, A\&A, 411, 131

[18] Vercellone et al. 2008, ApJ, 676L, 13

[19] Vercellone et al. 2009, ApJ, 690, 1018 